\title{
Le rugby à la croisée du management et de la communication
}

\section{Alexandre Eyries}

\section{(2) OpenEdition}

1 Journals

Édition électronique

URL : http://journals.openedition.org/communicationorganisation/4807

DOI : 10.4000/communicationorganisation.4807

ISBN : 979-10-300-0155-6

ISSN : 1775-3546

\section{Éditeur}

Presses universitaires de Bordeaux

\section{Édition imprimée}

Date de publication : 1 décembre 2014

Pagination : 249-264

ISBN : 978-2-86781-905-6

ISSN : 1168-5549

\section{Référence électronique}

Alexandre Eyries, "Le rugby à la croisée du management et de la communication », Communication et organisation [En ligne], 46 | 2014, mis en ligne le 01 décembre 2017, consulté le 26 avril 2019. URL : http://journals.openedition.org/communicationorganisation/4807 ; DOI : 10.4000/

communicationorganisation.4807 


\title{
Le rugby à la croisée du management et de la communication
}

\author{
Alexandre Eưries ${ }^{1}$
}

Cet article s'attachera - à partir d'une revue des travaux menés en communication et marketing politique (Maarek, 2007), communication organisationnelle (Jolivet, 1998), en sociologie du sport (Caillat, 1989 ; Brohm, 1992 ; Bromberger, 1995 ; Gasparini, 2000 ; Barbusse, 2002), en management et marketing sportif (Bouchet et Sobry, 2005 ; Bayle, 2007 ; Corneloup, 1999 ; Fontanel et Paturel, 2008) - à analyser la façon dont les valeurs sportives parviennent à dépasser le strict contexte du sport pour se voir investies de potentialités intéressantes par des hommes politiques et des chefs d'entreprise.

Comment un sport comme le rugby « contribue-t-il à fortifier l'identité d'une ville, d'une région, en une époque où les repères territoriaux et sociaux se font rares » (Sansot, 2005, p. 14) ainsi que l'écrit le philosophe et sociologue Pierre Sansot dans son ouvrage Le rugby est une fête, le tennis non plus ? Comment peut-il être le vecteur de la transmission d'un message plus largement humaniste ? Comment peut-il également être détourné de sa fonction première pour servir d'autres objectifs?

Nous nous efforcerons dans un premier temps d'analyser les valeurs spécifiques au rugby pour mettre en lumière celles qui pourraient faire l'objet d'une transmission en direction de la société civile et des sociétés privées et les modalités précises selon lesquelles cette transmission pourrait se dérouler. Nous étudierons dans un second temps la propension marquée des politiciens, des managers et des chefs d'entreprise à s'emparer d'évènements sportifs pour asseoir leur popularité, améliorer leur visibilité ou asseoir une politique commerciale. Nous nous intéresserons à leur manière de mettre en scène de manière ostentatoire leur connaissance d'un sport pour créer une dramaturgie, un véritable spectacle renforçant le sentiment de proximité avec le public venu assister au match, public qui représente, bien évidemment, une part non négligeable leur possible électorat. C'est d'ailleurs ce que note fort 
judicieusement l'anthropologue et sociologue Georges Balandier dans Le pouvoir sur scènes lorsqu'il écrit que la communication politique emprunte parfois au théâtre certains artifices destinés à surprendre l'auditoire : « La "théâtrocratie" [...] règle la vie quotidienne des hommes en collectivité : elle est le régime permanent qui s'impose aux régimes politiques divers, révocables, successifs » (Balandier, 1992, p. 13). Nous essaierons de montrer de manière plus approfondie que la mise en scène de la connaissance du rugby par les hommes politiques constitue une forme très visuelle d'activité rituelle, ainsi que le remarque Pascal Lardellier : « ce spectacle vivant et à représentation unique que constitue le rite, et dont les acteurs se trouvent être les plus hauts représentants de l'État, ne s'épuise pas en une hypothétique finalité de divertissement, $[\ldots]$ le rite politique constitue en fait un moment du pouvoir en performance, un accomplissement authentique de la vie institutionnelle. Lors de ces rites, le pouvoir se donne à voir à la société, et le fait de se montrer ainsi revêt une dimension politique active » (Lardellier, 2003, p. 168).

Les valeurs rugbystiques sont ainsi mises au service de dispositifs communicationnels élaborés où la vie politique, entrepreneuriale et le sport collectif sont appariés. Au-delà des pures considérations politiques et des différentes échéances électorales, il ne demeure que des rites structurants qui, du rugby à la société, contribuent sinon à fonder du moins à renforcer un vivre ensemble : " les rites sont partout présents, et ils remplissent d'inestimables fonctions sociales. [...] Ils produisent du lien, de la mémoire et de l'appartenance, tout en donnant un cadre esthétique à nos relations [...], sont tout à la fois des écrins pour nos relations et une architecture pour la société » (Lardellier, 2013, p. 13). Considérons à présent les valeurs spécifiques du rugby à l'aune de leur possible transmission à l'ensemble des individus et des salariés des organisations qui constituent le cœur d'une qui est en même temps une agora politique.

\section{Les valeurs du rugby : une valeur ajoutée pour la société et pour l'organisation?}

\section{Les valeurs humanistes du rugby}

Dans l'univers du rugby, les dimensions physique, technique et stratégique sont très présentes au cours de la partie mais n'occultent à aucun moment la richesse des relations interpersonnelles et intersubjectives (faites de confiance, de connaissance réciproque et de complémentarité) qui se tissent entre les quinze joueurs d'une même équipe. Ces relations sont nourries et soustendues par des valeurs qui en font tout l'intérêt et également toute la beauté.

Le rugby suppose dans un premier temps la coopération entre partenaires d'une même équipe. Mais cela n'empêche pas, bien au contraire, le respect d'autrui à travers les adversaires (on joue contre eux, mais aussi avec eux) : "l'affrontement avec l'autre permet donc de se situer, de trouver sa place. On se mesure à un adversaire égal, miroir de soi-même, pour affirmer sa 
propre identité. [...] Lidéal maçonnique disait "se construire ensemble", et c'est bien ce qui fait l'essence même du rugby " (Herrero, 2003, p. 13). Le combat collectif (notamment dans l'épreuve de force que constitue la mêlée) et les nombreuses situations frontales d'affrontement physique (dans les regroupements, par exemple) présupposent à la fois le courage individuel et la solidarité face à l'adversité. Pierre Sansot écrit à ce propos : « le rassemblement d'un groupe autour d'une action fondamentale [...] touche aux confins du sacré, au tréfonds de la condition humaine " (Sansot, 2005, p. 40-41). Cela suppose donc d'accepter le choc et la chute pour démarquer un partenaire, conserver ou recouvrer la possession de la balle.

La pratique du rugby s'accompagne également d'intelligence tactique pour choisir les solutions individuelles et collectives les plus adaptées au rapport d'opposition mis en évidence par le contexte de jeu. En effet, le « bon joueur doit capter le plus grand nombre d'informations [...] et opérer le "bon choix". [...] Ce qui rend la performance méritoire, c'est qu'il possède un délai fort court. [...] De là une vivacité d'esprit indispensable » (Ibid., p. 25-26). Cette intelligence tactique s'accompagne aussi d'une nécessaire prise d'initiative pour sortir à bon escient des schémas de jeu préétablis et impulser un peu de créativité. Celle-ci consiste à aller " du désordre à l'ordre, ou, à l'inverse, de l'ordre au désordre, bref à restructurer l'ordre [...] de la relation à autrui [...] d'une manière nouvelle qui nous est plus favorable» (Ibid., p. 26). Le rugby, sport collectif de combat indissociable d'une certaine rudesse place au firmament le goût de l'effort qui permet de supporter la durée des matches, les aléas du score et des intempéries. Enfin, la dernière de ces valeurs - et non la moindre - est la convivialité qui se manifeste après le match lors de la fameuse "troisième mi-temps" et permet de poursuivre-ainsi que l'observe Jean-Jacques Boutaud - la rencontre des autres dans le cadre festif et décontracté d'un repas : « la table rassemble, réunit, place au même niveau, gomme les barrières sociales. Elle installe et instaure le partage, la confiance, voire la confidence. [...] Elle est signe de communauté, de communion " (Boutaud, 2011, p. 17). Si pour le stratège Carl von Clausewitz, la guerre est la continuation de la politique par d'autres moyens, la troisième mi-temps constitue au rugby à la fois la continuation rassérénée des percussions rugueuses et l'instrument permettant l'instauration d'une paix des braves qui prolonge sur le plan de la parole la geste guerrière des corps. Ainsi, la table autour de laquelle se réunissent les joueurs des deux équipes rassemble un microcosme organisé, une véritable microsociété et représente " un lieu de plaisirs partagés, de communion des corps et des cœurs " (Lardellier, 2011, p. 23). La troisième mi-temps au rugby est organisée autour de la commensalité, c'est-à-dire du repas pris en commun : à ce titre, elle sera considérée, écrit Pascal Lardellier, « comme le cœur de la vie sociale et un formidable théâtre relationnel, lieu de partage et de plaisirs, impitoyable prisme sociologique » (Ibid., p. 31). Tout à la fois art du partage, plaisir de l'échange et volupté de la transmission, le 
rugby est une " métaphore vive " (pour faire référence à Paul Ricœur) de la société française. Il fonctionne comme un puissant révélateur auquel les pratiques humaines sont passées.

\section{Les valeurs ajoutées}

Comment les valeurs du rugby peuvent-elles être étendues à d'autres contextes que celui du sport ? En quoi peuvent-elles être éclairantes pour les sociétés en général en tant que communautés régies par des règles) et pour les organisations? En quoi les valeurs issues d'un sport de combat pourraientelles être profitables à l'ensemble de la classe politique ?

Nous proposons ici de montrer en quoi les valeurs du rugby peuvent précisément constituer un apport enrichissant pour les relations humaines qui s'organisent autour " du rêve, du rire, de l'émotion, des joies communes " (Sansot, op. cit., p. 15) qui fournissent la trame de la vie en collectivité. J'analyserai d'abord les relations structurant la vie politique autour d'un débat d'idées qui met en présence deux partis antagonistes (comme le font les équipes de rugby qui rentrent chaque fin de semaine sur un terrain pour s'y affronter), puis les logiques convergentes qui peuvent exister entre le rugby et le monde de l'entreprise.

Si le rugby est né d'une transgression (saisir la balle à la main et la porter dans l'embut) - comme toutes les actions politiques décisives (il n'y a qu'à penser à l'appel du 18 juin lancé par le Général de Gaulle et s'inscrivant à contre-courant du discours de Pétain) - il existe néanmoins des différences importantes entre ce sport collectif et la politique, ainsi que l'observe avec justesse le journaliste Thomas Legrand : « les qualités pour jouer au rugby sont celles dont on rêve pour la politique : il faut être courageux, collectif, loyal et ne pas avoir un ego surdimensionné » (Legrand, 2011). En effet, la communication politique prend parfois l'allure d'une joute oratoire entre deux politiciens qui parviennent mal à cacher leur volonté de puissance et leur désir de reconnaissance. La guerre des chefs - pour le moins triviale - qui a opposé Jean-François Copé et François Fillon entre avril et mai 2013 - dans le cadre des élections organisées pour briguer la présidence de l'UMP - est là pour en témoigner. Une différence fondamentale entre le rugby et la vie politique se situe également au niveau de la mentalité spécifique à chaque activité : en effet « quand on porte la balle, au rugby, c'est généralement pour essayer de fixer le plus d'adversaires sur soi afin d'offrir le ballon à un partenaire qui aura ainsi la voie plus libre. C'est pour lui que l'on aura avancé et mobilisé le plus possible la défense adverse. » (Legrand, 2011).

Au rugby les actions des huit avants (notamment au cours d'une mêlée) servent à faire briller les sept arrières, alors qu'au contraire tout ce qu'entreprend un homme politique c'est pour se mettre lui-même en lumière. Bien plus qu'un simple sport collectif comme il y en a tant (football, handball, basket-ball), le rugby se singularise par sa conception éminemment altruiste du jeu : sans les 
autres membres de l'équipe au rugby on ne peut rien faire : « les rares vedettes de l'histoire du rugby: Spanghero, Rive ou Chabal le sont plus par leur dégaine que par leurs performances. Il n'y a pas de stars dans le rugby tout simplement parce qu'il est très difficile » (Legrand, 2011), impossible même d'être mis en évidence au détriment de ses coéquipiers. Comme l'écrit Pierre Sansot dans son ouvrage Le rugby est une fête, " il y a au rugby, de par sa tradition, de par la rudesse de son jeu, un goût de la simplicité, un respect de la vérité » (Sansot, 2005 , p. 21) qui bat en brèche toute tentative de mise en scène de soi, toute propension au paraître qui semble, au contraire, constitutive de l'exercice de la politique. Mais ces différences certes importantes ne doivent pas occulter une réalité partagée, la nécessité commune à ces deux univers de mobiliser « un grand sens stratégique, il faut être malin mais pas trop retors » (Legrand, 2011). Au rugby tout comme en politique, il faut savoir alterner entre des phases de confrontation directe et des phases d'évitement, entre la lutte acharnée et une certaine forme de prise de recul par rapport à l'effervescence ambiante. Il y a cependant une similitude profonde entre le rugby et la politique, c'est cette capacité à s'engager corps et âme dans un pugilat qui peut être d'une rare violence, et à revenir immédiatement après à une attitude pacifique sitôt le coup de sifflet final donné.

À la fin d'un match de rugby, il n'est pas rare de voir de solides gaillards « la gueule ensanglantée et les oreilles en choux fleurs, faire des haies d'honneur à leurs adversaires en retournant au vestiaire » (Legrand, 2011). Dans le champ de la politique ainsi que des campagnes et stratégies de communication qui en sont indissociables, l'opinion publique a tendance à ne retenir que des rivalités soigneusement entretenues à grands renforts de joutes médiatiques qui constituent un aperçu biaisé et réducteur de la situation : " en réalité ces hommes et ces femmes qui s'entredéchirent savent mieux que d'autres passer outre, "jeter la rancune à la rivière" comme disait Giscard »(Ibid.). Le déroulement et l'issue de la primaire socialiste avant même le début de la campagne présidentielle 2012 ont donné lieu à des séquences dignes des plus belles images de fairplay au rugby. Durant la même campagne, Nicolas Sarkozy n'a eu de cesse de vouloir rallier à sa cause ceux qui l'avaient sévèrement critiqué (Villepin, Hulot, Morin, etc.), adoptant une attitude rugbystique très courante, celle qui consiste "à serrer la main de celui avec lequel on vient de s'échanger quelques marrons »(Ibid.). Néanmoins, il reste une partie du public concerné par la politique (celui des militants et des sympathisants) qui devrait calquer leur comportement sur celui des joueurs de rugby. Durant un match de rugby, les supporters se chambrent gentiment et se mélangent avec plaisir dans les tribunes. Le rugby est un combat passionnant parce que dépassionné, un exutoire et une joute pacifique où la violence est neutralisée.

$\mathrm{Au}$ sein de l'entreprise, une introduction des valeurs du rugby dans la réalité des expériences managériales et des pratiques entrepreneuriales est possible, mais sous certaines conditions. La première est que le management 
rugbystique d'une entreprise ne peut fonctionner qu'avec une équipe travaillant dans la longévité et dans la stabilité (le contexte de précarité généralisée que nous connaissons actuellement dans les organisations ne s'y prête absolument pas). La seconde est que ce nouveau modèle managérial, pour avoir toutes les chances de réussir, doit être pris en charge conjointement par la direction de l'entreprise et par le service communication qui a vocation à faire connaître ces initiatives, à les médiatiser et à en amplifier la portée.

Qu'est-ce que les entreprises ont à gagner en adoptant ce modèle venu du rugby?

Le rugby est en premier lieu porteur d'un modèle particulier et original de gouvernance qui impose, comme le remarque François Jolivet, « que chacun soit professionnel, joue son rôle, dans l'adversité, comme au rugby, mais aussi que chacun puisse dialoguer après la sortie des vestiaires ou lors de la troisième mi-temps. Il faut un esprit constructif et que chacun " (Jolivet, 1998) puisse à tour de rôle aussi bien perdre que gagner et, plus encore, se construire aussi bien dans l'adversité et la difficulté que dans la considération pour l'autre.

Selon Emmanuel Bayle, dans le rugby comme dans l'entreprise, certaines logiques existent qui s'appuient sur les "principes générateurs de l'action individuelle et collective en situation : les valeurs (culture), les influences sur autrui (pouvoir), les intérêts (stratégie)" (Bayle, 2007, p. 65). Comme le démontre Béatrice Barbusse, il existe de profondes similitudes de fonctionnement entre la vie d'une entreprise et celle d'un club de rugby. Jocelyn de Just, consultant en management, souligne à son tour les importants efforts consentis par le monde de l'entreprise pour faire siennes les valeurs humanistes du rugby. Cette assimilation voulue passe préalablement par l'établissement d'un univers de référence commun ou, à tout le moins, par une estompe des différences entre les deux écosystèmes. Au rugby, la solidarité aide à « se remettre en question, afin de trouver des solutions ensemble » (De Just, 2011). L'humilité est de rigueur, c'est : «le maillot [qui] est valorisé, pas le joueur » (Ibid.). Avant le match, le coach rappelle à ses joueurs leur importance dans le système de jeu et ce qu'il attend d'eux. L'important pour une équipe (ou une entreprise) c'est la force de son collectif : « on est parfois amené à souffrir sur un dossier, à surmonter une réelle difficulté pour boucler un projet. Mais si on fait corps avec les copains, on sait qu'on va y arriver » (De Just, 2011). Pour motiver ses employés, un manager peut raconter l'histoire d'un succès ou d'une victoire " pour inspirer les collaborateurs " (Ibid.). Si un manager " délègue des objectifs » à l'autre (De Just, 2011.), il établira avec lui une relation de confiance et essaiera de créer une culture commune « composée de valeurs, de normes, de rites, de mythes et de héros dont la vocation est de créer une communauté »(Barbusse, 2002, p. 409). 
De quelle manière l'entreprise transpose-t-elle dans ses activités managériales des pratiques issues du rugby?

L'entreprise s'inspire en premier lieu de la façon spécifique qu'ont les rugbymen de gérer le stress lié à la compétition et aux matches à enjeux et de le transformer en source de motivation : "Areva, Total, Lapeyre ou Stéria ont ainsi fait appel à des formations utilisant les règles de jeu du ballon ovale pour réussir certains projets : ouverture à l'international, amélioration de la qualité ou résolution de conflits. Ici, la mêlée, les techniques de plaquage ou les jeux de touche permettent aux salariés de tester et de renforcer leur cohésion. Et à chaque membre de l'équipe de trouver sa place ${ }^{2} »$.

Nous aborderons à présent le cas plus précis d'une entreprise créant des logiciels spécialisée dans le marketing à la performance qui s'appelle PublicIdées et dont le pôle technique est situé à Bordeaux. Cette entreprise s'est inspirée à partir de 2011, d'une pratique très répandue de nos jours dans le rugby de l'hémisphère sud, une méthode de travail s'appuyant sur les skills à savoir le travail de certains gestes techniques pour renforcer l'habileté et la maîtrise du geste. L'entreprise Public-Idées a donc entrepris un travail par cycles itératifs courts qui vise à répondre aux mieux aux exigences du client. Ce n'est d'ailleurs pas anodine si l'entreprise s'est pour ce faire appuyée sur une méthode baptisée "Scrum », du nom de la mêlée qui constitue la pierre angulaire du rugby. Comme dans la mêlée au rugby, l'accent est mis sur les interactions entre les personnes beaucoup plus que sur les processus : au rugby, la mêlée n'est forte que lorsqu'il y a communion des huit avants qui ne font qu'un pendant l'épreuve de force. Lidée du directeur technique est de satisfaire le client qui est au cœur du jeu et de mobiliser dans l'action ses salariés. L'entreprise doit être soudée comme une équipe de rugby, travailler collectivement, et s'adapter en permanence aux aléas (non pas du jeu) mais des étapes émaillant la réalisation d'un produit. Au rugby, des phases de jeu différentes s'enchaînent, tour à tour dynamiques (actions de trois-quarts) ou statiques (phases de conquête). Dans l'entreprise Public-Idées, chaque phase, dénommé «sprint» s'attache à créer " une première version du produit qui sera enrichie d'une V2 [...]. Deux autres termes sont essentiels : "product owner" (PO), l'œil du client [sur] le projet, [...] et "scrum master" [... $]^{3}$ » ou garant de la mêlée c'est-à-dire en l'occurrence de la réalisation d'un logiciel. Ce rôle est identique à celui que tient le talonneur dans la mêlée, il fixe le cap et assure la stabilité de l'édifice. Suivent ensuite " la transparence " et "l'inspection " pour vérifier que les travaux sont sur le droit chemin, dont les équivalents

2 Adrien Cahuzac, "Motiver par le rugby ", L’usine nouvelle, n 3250, 9 septembre 2011, http://www. usinenouvelle.com/article/motiver-par-le-rugby.N157469

3 Marie-Madeleine Sève, « Le rugby comme modèle... de management innovant », 23 avril 2014, http:// www.latribune.fr/entreprises-finance/20140422trib000826493/le-rugby-comme-modele-de-managementinnovant.html 
sont les séances de décryptage vidéo des séquences de jeu après le match. Au terme de ces débriefings, les salariés de l'entreprise Public-Idées découvrent un nouvel état d'esprit reposant sur la prise d'initiative et la responsabilisation. Comme au rugby, chacun s'approprie le projet tout en restant libre de faire ses propres expériences, de prendre des risques et d'émettre des avis personnels. C'est le groupe qui dresse sa propre autocritique et pointe des perspectives d'amélioration, décide des directions dans lequel on se doit d'aller. L'avantage d'un management transposé du monde du rugby repose précisément sur cette double dynamique : d'une part le travail collectif visant à mobiliser toute une équipe, et d'autre part la liberté offerte à chacun de tenter, de proposer de nouvelles choses, des nuances ou des infléchissements : " Chacun doit donc apprendre à [s'] écouter et dire son opinion avant que le groupe se mette d'accord, par vote ou consentement. Et le partage comme le feed-back positif doivent devenir un réflexe ${ }^{4} »$.

Au final, pour cette entreprise, travailler de cette manière en s'inspirant des pratiques du rugby a présenté l'avantage considérable de gagner en rapidité, de renforcer la coopération au sein des membres de l'équipe, d'encourager la responsabilisation et l'auto-organisation et de rendre la transmission de l'information plus fluide, comme doit l'être celle de la balle au rugby.

Mais pour éclairante qu'elle soit, cette étude de cas doit être néanmoins nuancée et relativisée, car il n'y a rien de plus complexe que les interactions interpersonnelles et que la gestion de l'émotion et des affects au travail. Si elle a pu porter ses fruits pour l'entreprise Public-Idées et permettre à son activité de se développer considérablement et d'être beaucoup plus efficace, il faut néanmoins se garder, en matière de management par le rugby de tout angélisme et ne pas mésestimer un autre type de récupération, managérial celui-là : "Quand on connaît la capacité fédératrice et identificatoire du sport, on comprend pourquoi les entreprises qui souhaitent fédérer leurs salariés autour de valeurs communes se tournent vers le sport»(Barbusse, op. cit., p. 409).

La communication managériale et politique s'est inscrite depuis quelque temps dans une dynamique d'acculturation visant à s'approprier certaines valeurs humaines constitutives du rugby, mais elle a tôt fait de les sacrifier sur l'autel de la performance économique et théâtrale.

\section{Une transmission biaisée : la récupération politique et marchande des valeurs du rugby?}

\section{La récupération politique du rugby}

Au moment où la population française est confrontée à des défis sans précédent (crise sociétale, perte d'intérêt pour la politique, difficultés

4 Marie-Madeleine Sève, "Le rugby comme modèle... de management innovant ", 23 avril 2014, http:// www.latribune.fr/entreprises-finance/20140422trib000826493/le-rugby-comme-modele-de-managementinnovant.html 
économiques, etc.),ilestévident que s'intéresser au rugby sousl'angle des valeurs peut sembler, sinon vain, du moins en rupture totale avec les préoccupations de l'opinion publique. On pourra tout d'abord expliquer « l'engouement porté au rugby par des raisons mineures, voire dérisoires : les hommes gaspillent leur enthousiasme et se détournent, en revanche, de ce qui devrait susciter leur ferveur " (Sansot, op. cit., p. 32) en l'occurrence les arcanes secrets de la vie de la communauté nationale. On pourra aussi percevoir le rugby, le football et bien d'autres sports comme des jeux sans malice ni enjeux véritables. On y verra, enfin, d'agréables passe-temps auxquels recourent celles et ceux qui y voient une forme de divertissement au sens pascalien du terme, une échappatoire par rapport à un quotidien parfois morose. La réalité est somme toute assez différente. En effet, il faut bien, comme l'écrit Pierre Sansot dans l'ouvrage déjà cité, donner une orientation à la vie et " ordonner un calendrier un peu plus motivant que celui de notre travail » (Sansot, op. cit., p. 33). Quand la vie de tous les jours oscille entre la stagnation et l'emballement, entre les petits tracas et les grandes joies, alors nous devons - selon Pierre Sansot - trouver de multiples occasions de nous rassembler autour de représentations et de passions communes : " la joie d'être ensemble n'est pas, non plus négligeable, s'il est vrai que nous n'avons pas beaucoup d'occasions de nous rassembler et de vibrer collectivement» (Ibid., p. 33).

Quel meilleur dénominateur commun entre le rugby et la politique que les vecteurs de rassemblement, les activités permettant aux hommes de se retrouver autour d'émotions partagées par le plus grand nombre?

Les hommes et les femmes politiques l'ont bien compris qui ont été prompts à s'emparer des grands évènements sportifs pour en faire des moments obligés de leur activité dans l'espace public. Ils ont rapidement saisi la part dramatique que recelaient les grandes compétitions sportives et les ont intégrés dans leurs interventions adressées à la collectivité. Pascal Lardellier analyse ces interventions comme des moments particuliers de la vie en société : « la "représentation du Corps collectif" est une image globale, une impression générale que va produire d'elle-même la communauté. [...] Cette image collective, caractérisée par un fort principe d'idéalisation [...] sera perçue dans le regard de [l'autre], la construction du dispositif rituel étant toujours spéculaire, c'est-à-dire conçue en miroir » (Lardellier, 2003, p. 210). Ainsi, pour un leader politique, un match de rugby constitue une représentation du corps collectif de la France, mais plus encore un moyen de créer autour du match une dramaturgie incluant le public qui représente une frange précise de son électorat potentiel. Georges Balandier l'avait analysé dès 1992 : « tout système de pouvoir est un dispositif destiné à produire des effets [...] qui se comparent aux illusions créées par la machinerie du théâtre »(Balandier, op. cit., p. 14). C'est ce qu'a remarquablement analysé le journaliste politique et essayiste Alain Duhamel dans une tribune publiée le 12 septembre 2007 sur le site du journal Libération: " le rugby n'est pas seulement l'un des sports les plus pittoresques 
et les plus virils (souvent aussi l'un des plus brouillons), c'est également, c'est nécessairement, c'est de plus en plus un objet politique identifiable, parce qu'il y a un usage politique du rugby et qu'il y a, que cela fasse plaisir ou non, une nature politique du rugby, presque une vocation politique ${ }^{5} »$.

Ce n'est pas pour rien que la Coupe du Monde de Rugby 2007 - organisée en France pour la première fois en six éditions - a constitué un évènement sans précédent à la fois sur le plan social et sociétal, économique, et bien évidemment politique en dépit, pour le XV de France, de résultats sportifs inégaux ${ }^{6}$. La communication politique du Président de la République alors en exercice (Nicolas Sarkozy) a largement contribué à faire de cette compétition une incarnation de la spectacularisation de la vie politique, ainsi que l'a analysé l'anthropologue Georges Balandier : « le pouvoir acquis est théâtral dans l'acception la plus immédiate du mot » (Balandier, op. cit., p. 15). La prise en charge - théâtrale - par le pouvoir politique d'un évènement sportif au potentiel dramatique certain participe entièrement d'une démarche de récupération des valeurs du sport en général et du rugby en particulier. Certains indicateurs (la place spectaculaire et la surexposition que lui offrent les médias, les remarquables taux d'écoute des téléspectateurs) ne trompent pas : «le public frémit, les Français rêvent de reproduire sous Nicolas Sarkozy avec le ballon ovale ce qu'ils avaient vécu sous Jacques Chirac avec le ballon rond, l'Hexagone soudain métamorphosé en une nation de supporters, avec une envie collective de vibrer, de gronder, de vociférer, d'ovationner les siens, de triompher tous ensemble par le truchement hypothétique de quinze béliers soudain déchaînés " (Duhamel, 2012) La compétition rugbystique devient ainsi un moyen de prendre le pouls de la population française et de l'entendre battre à l'unisson au gré des heurs et des malheurs de ses héros.

La farouche volonté affichée par Nicolas Sarkozy - qui était alors un Président de la République fraîchement élu - de se poser en fin connaisseur d'un sport complexe tel que le rugby s'inscrit dans la continuation de la campagne présidentielle qui l'a conduit au plus haut sommet de l'Etat. En effet, en ayant laissé entendre qu'il était depuis toujours un amateur passionné de rugby, Nicolas Sarkozy a usé et abusé d'une narration utile qui lui a, jusque-là, parfaitement réussi : le storytelling que Christian Salmon définit comme un " art de raconter les histoires " (Salmon, 2008, p. 7). En cela, il s'est montre doué pour exploiter et récupérer habilement le « vouloir-vaincre ensemble qui saisit, hystérise parfois le pays, comme si le sport et la compétition mondiale devenaient une affirmation patriotique de soi, un drapeau d'espérance ou de revanche, un substitut du destin, un peuple fugitivement réuni par une horde de colosses en bleu et boue » (Duhamel, 2012). En praticien avisé du

5 Alain Duhamel, 12 septembre 2007, http://www.liberation.fr/tribune/2007/09/12/rugby-objet-politiqueidentifiable_101575

6 Le XV de France a été battu 17-12 le 7 septembre 2007 (en match d'ouverture de la Coupe du Monde) sans aucune gloire ni panache par des Argentins beaucoup trop rusés. 
storytelling, Nicolas Sarkozy s'est préparé, si les dieux des stades étaient avec lui, à se transformer en supporter suprême, faisant corps avec l'équipe victorieuse, soutenant ses efforts, partageant ses épreuves, ses blessures et surtout ses lauriers, bénéficiant de sa popularité extatique, cela va de soi, à condition de savoir chavirer quand il le faut dans un populisme savamment dosé. Car c'est bien évidemment de cela qu'il s'agissait pour l'homme politique : de profiter un maximum de la réussite de l'équipe nationale (en l'occurrence de rugby), de s'attribuer les victoires et les exploits des joueurs en espérant que leurs moments de gloire - les fameuses quinze minutes de célébrité dont a parlé Andy Warhol - rejaillissent sur ses épaules. C'est ce que Pascal Lardellier appelle 1' " appar-être " (Lardellier, 2003, p. 203) et qui se situe à la croisée entre une image soi-disant naturelle et une image construite. Cela ressortit également au mécanisme bien rodé du marketing politique que Philippe J. Maarek a analysé comme « une démarche globale de conception, de rationalisation et d'accomplissement de la communication politique moderne ». (Maarek, 2007, p. 3). La stratégie de communication était bien connue et avait déjà été utilisée avec succès par Jacques Chirac qui, en 1998, durant le Mondial de football, alors qu'il n'avait jusqu'alors montré que peu d'intérêt pour le sport, "s'était réincarné en mascotte du onze tricolore " (Duhamel, 2012). Nicolas Sarkozy a saisi au vol l'occasion de s'emparer d'une manifestation capable d'émouvoir le peuple. C'est d'ailleurs ce qu'écrit Alain Duhamel, remarquable scrutateur des mœurs politiques françaises : «Nicolas Sarkozy, adepte du jogging, du vélo, du tennis, fervent supporter du PSG, se recycle en toute hâte, apprenant d'urgence l'alphabet et la grammaire du rugby. Si le XV tricolore se redresse et retrouve à temps la furia francese, le Président sera fin prêt pour devenir quelques semaines l'emblème du bonheur français. Sinon, il mènera le deuil face à l'injuste fatalité. Pourquoi ne pas instrumentaliser le rugby après le football ? " (Duhamel, 2012).

On saisit bien à lire Alain Duhamel tout l'intérêt que Nicolas Sarkozy a eu à reprendre à peu de frais l'engouement généré par l'enchaînement des matches de rugby de très haut niveau, par la succession des rencontres à élimination directe qui génèrent de la curiosité, du suspense et de la surprise et soulignent ainsi la «dimension communicationnelle des émotions" (Baroni, 2007, p. 20) analysée par Raphaël Baroni. Il a su avec intelligence mettre en parallèle dans ses discours la trajectoire superbe mais difficile des rugbymen français durant la Coupe du Monde 2011 et sa propre situation inconfortable au sommet de l'État quelques années après, ainsi que l'analyse le journaliste Hervé Gattegno dans un article publié dans le Point en décembre 2011 : «N. Sarkozy a toutes les raisons de succomber à une nouvelle passion pour le rugby. La performance du XV de France en Coupe du monde a de quoi l'inspirer. Pour la vaillance qu'ont montrée les Français, à qui les pronostiqueurs promettaient une raclée monumentale, et que personne n'imaginait en finale il y a trois semaines. On voit bien le parallèle : N. Sarkozy est donné battu dans tous les sondages, son camp est 
divisé, ses partisans assez démobilisés et lui-même a l'air d'avoir perdu la main » (Gattegno, 2011). Nul doute que le Président de l'époque aura préféré retenir l'image d'une équipe qu'on disait moribonde et qui a échoué d'un point face à la meilleure équipe du monde (les All Blacks) au terme d'une farouche bataille, gagnant ainsi le respect du Monde du rugby. Sa communication à propos des valeurs du rugby, démagogique et utilitariste, constitue un modèle de récupération politique d'un évènement d'intérêt collectif mais il en existe une autre, plus larvée mais tout aussi efficace, l'instrumentalisation et la marchandisation du sport.

\section{La récupération marchande du rugby}

Depuis le passage du rugby à l'ère professionnelle en 1995, un certain nombre d'alliances avec le monde de l'entreprise se sont opérées, d'abord de manière relativement timide et balbutiante, puis de façon plus affirmée et davantage institutionnalisée. Mais l'histoire débute en réalité bien plus tôt, au début du $\mathrm{XX}^{\mathrm{e}}$ siècle, dans la région du Massif Central. L'existence du club de l'ASM Clermont Auvergne est fortement liée à l'entreprise dominante de la ville de Clermont-Ferrand, à savoir la manufacture Michelin, qui a fait de gros investissements pour se doter d'installations sportives omnisport.

C'est en 1911 que la manufacture Michelin fonde l'Association Sportive Michelin (ASM), qui a pour but de divertir les employés des usines clermontoises. C'est un club omnisport qui possède une équipe de rugby, mais aussi des sections football, basket-ball ou encore athlétisme. Cependant, en 1922, l'ASM doit changer de nom pour se conformer à une directive de l'Union des sociétés françaises de sports athlétiques (USFSA) qui interdit aux équipes de posséder le nom d'une entreprise dans leur nom, afin de lutter contre la publicité et la concurrence déloyale. L'entreprise Michelin décida alors de changer l'Association Sportive Michelin en Association Sportive Montferrandaise, Montferrand étant le nom d'un quartier de Clermont où se situaient à l'époque les installations du club. Aujourd'hui, l'ASM existe toujours et a été rebaptisée ASM - Clermont Auvergne en 2004. Le mécénat sportif de Michelin ne représente plus que $11 \%$ du budget du club, mais ce dernier joue toujours au stade Marcel-Michelin. Ce premier niveau d'interpénétration entre les pratiques sportive et entrepreneuriale correspond à une recherche de performance sportive qui transforme les objectifs, les bases de gestion, la structure des clubs. Comme l'écrivent Gautier Fontanel et Robert Paturel : « leurs résultats sportifs, mais aussi leur niveau de compétition, sont sources de financement, de notoriété et de soutien populaire. La performance sportive est recherchée, ce qui implique un investissement plus important en termes sportifs, financiers ou de temps » (Fontanel et Paturel, 2008, p. 4).

À l'époque où le rugby n'était pas encore professionnel, certains joueurs ont été pionniers en matière de revenus dérivés de leurs pratiques sportives. 
Je songe notamment aux frères Spanghero (Laurent et Claude), premiers rugbymen français à avoir acquis une certaine célébrité sportive à partir de laquelle des entreprises ont bien voulu investir et grâce à laquelle, en retour, ils ont pu créer dans les années 1970 leurs propres sociétés, d'abord de location de voitures (mais elle a rapidement cessé son activité), puis de préparations alimentaires à base de viande. C'est précisément cette société qui a été frappée par un violent scandale sanitaire impulsé par le remplacement de la viande de bœuf par de la viande de cheval dans certains plats cuisinés ${ }^{7}$. On touche là aux limites des rapprochements entre rugby et entreprise, lorsque les rugbymen servent de prête-nom, d'hommes-sandwiches et de garants de la moralité à certaines pratiques entrepreneuriales. Il s'agit là d'un premier niveau d'instrumentalisation ou de récupération du rugby.

Mais il en existe d'autres. Beaucoup plus près de nous d'un point de vue temporel, les entreprises sont nombreuses à pratiquer le sponsoring, notamment sous la forme de contenu publicitaire figurant sur les maillots des joueurs. Nous nous concentrerons plus particulièrement sur trois entreprises qui ont noué des partenariats avec des clubs de rugby ou avec la Fédération Française de Rugby par exemple à l'occasion d'une Coupe du Monde ou de la retransmission des matches du Tournoi des Six Nations. La Société Générale, en s'appuyant sur des valeurs partagées (respect, esprit d'équipe, engagement, convivialité), mène depuis vingt-sept ans des actions en faveur du rugby et se comporte comme un " un partenaire fidèle et impliqué, présent sur tous les terrains, qui s'attache à développer la pratique comme à soutenir le haut niveau ${ }^{8} "$.

Autre partenaire de longue date du rugby français, la Garantie Mutuelle des Fonctionnaires (GMF) « est le partenaire historique de la FFR depuis plus de 25 ans, mais elle apporte son soutien à de nombreuses associations sportives, petites et grandes qui ont en commun la passion du rugby et partagent avec elles des valeurs telles que la solidarité, la cohésion d'équipe et l'engagement ${ }^{9}$ ». La dernière entreprise qui a partie liée avec le rugby, Cap Gemini, se définit avant tout comme « une société d'hommes [dont] les principes fondateurs [...] ont été établis de manière à correspondre à un ensemble de valeurs inspirées [...] du rugby : honnêteté, audace, confiance, liberté, solidarité, simplicité et plaisir, [...] [responsabilité et indépendance], [...] [esprit d'équipe et de compétition], $[\ldots]$ [patience et respect] ${ }^{10} »$.

Ces trois entreprises n'investissent pas bien évidemment de manière entièrement philantropique mais bien parce qu'elles exigent en retour de la rentabilité et des retombées positives en termes d'image. Il est évident que le principal sponsor maillot (l'entreprise varoise Pizzorno) d'une équipe venant

7 Pascal Lardellier y a consacré un ouvrage paru en 2013 aux Éditions EMS, Risques, rites et plaisirs alimentaires. $8 \mathrm{http} / / /$ www.societegenerale.com/fr/connaitre-notre-entreprise/engagement/sponsoring-sportif/rugby 9 http://www.assurement-rugby.com/

10 http://www.fr.capgemini.com/a-propos-de-capgemini/groupe/un-socle-de-valeurs-communes 
de gagner la coupe d'Europe de Rugby (comme cela a été et comme c'est toujours le cas), en l'occurence le Rugby Club Toulonnais n'en retire que des avantages. D'abord, il est associé à une forme d'audace, à un certain esprit de conquête et d'entreprise qui se voient investis d'une aura symbolique. Ensuite, il ressort nécessairement grandi et renforcé d'un partenariat gagnant-gagnant. Quelle est alors dans cet univers la place d'un sport collectif comme le rugby qui situe le collectif au-dessus de tout?

La place la moins enviable, celle qui résulte d'une instrumentalisation. Comme l'écrit Béatrice Barbusse, les managers assimilent ce sport à certaines vertus (combativité, courage, loyauté, responsabilité, esprit d'équipe) qui « correspondent aux besoins de l'entreprise d'aujourd'hui. Dans un contexte de plus en plus concurrentiel où la performance est l'objet d'un véritable culte, la référence au sport (le domaine par excellence de la performance) s'impose comme une évidence pour tous les managers " (Barbusse, 2002, p. 407). Le sport est ainsi perçu un formidable vecteur d'assimilation des valeurs propre au système qui a contribué à l'engendrer, c'est-à-dire, écrit Béatrice Barbusse, que « la pratique sportive facilite l'adaptation à la société compétitive, à ses cadences, à son organisation » (Ibid., p. 408). Cela montre bien que dans ce genre de situation, l'intérêt affiché par une entreprise pour un sport comme le rugby reste circonscrit à un objectif de rentabilité et d'augmentation de la productivité, ce qui constitue pour le rugby l'instrumentalisation suprême tant pendant très longtemps ce sport s'est tenu éloigné des logiques comptables et financières.

Si les univers de l'entreprise, de la politique et celui du rugby semblent très éloignés les uns des autres, il y a en réalité quelques rapprochements possibles entre les trois domaines. Si les valeurs du rugby sont des exemples à suivre pour la collectivité, pour la vie de la cité au sens étymologique de la polis grecque) ou de l'organisation, elles sont en revanche en total décalage avec les us et les pratiques actuelles de la politique politicienne. Le rugby porte des valeurs positives, humanistes et fédératrices. Il promeut un modèle de société où l'affrontement entre deux équipes est viril mais correct, la violence étant neutralisée par le règlement qui encadre les phases de jeu, et où l'on est contraint de faire face, à n'importe quel prix et quelle que soit l'âpreté de l'opposition. Les hommes politiques en France ont rapidement infléchi leur communication vers le rugby générant une ferveur médiatique à laquelle ils adossent leur mise en scène du politique. Le rugby et l'entreprise ont élaboré au cours de cette dernière décennie des rapprochements dans un souci commun de recherche de la performance et ont, de ce point de vue seulement, des logiques convergentes. Toutefois, en important progressivement des modalités de gestion similaires à celles de l'entreprise, il s'agit pour la sphère sportive de rester sur le chemin de la performance sportive et organisationnelle alors que pour l'entreprise, lorsqu'elle instrumentalise le sport, il s'agit de pérenniser avant tout une performance économique. 
Rugby, politique et entreprise convergent bien vers une commune logique de performance, mais c'est la nature de la performance recherchée qui les distingue encore profondément. Si, l'orgueil et la volonté peuvent faire déplacer des montagnes aux rugbymen, il n'en faut pas plus aux chefs politiques et d'entreprise pour qu'ils franchissent le Rubicon et récupérent à peu de frais les valeurs d'un sport qu'ils méconnaissent si bien.

\section{BIBLIOGRAPHIE}

BALANDIER G., Le pouvoir sur scènes, Paris, Éditions Balland : collection Fondements, 1992.

BARBUSSE B., "Sport et entreprise : des logiques convergentes ? ", Paris, PUF, L'année sociologique, $\mathrm{n}^{\circ} 52$, p. 391 à 415.

BARONI R., La Tension narrative : suspense, curiosité et surprise, Paris, Éditions du Seuil : collection Poétique, 2007.

BAYLE E., "Essai de définition du management des organisations sportives : objet, champ, niveaux d'analyse et spécificité des pratiques managériales », Staps $\mathrm{n}^{\circ} 75$, Bruxelles, De Boeck Université, 2007, p. 59 à 81.

FONTANEL G., et PATUREL R., « La création d'une société sportive constitue-telle une pratique de l'entrepreneuriat ?", $2^{\mathrm{e}}$ Journées Georges Doriot, HEC Paris, 15 et 16 mai 2008.

HERRERO D., Dictionnaire amoureux du rugby, Paris, Plon, 2003.

JOLIVET F., "Management par projet : communiquer et après ? ", http:// communicationorganisation.revues.org/2050

LARDELLIER P., Théorie du lien rituel. Anthropologie et Communication, Paris, L'Harmmattan : collection Communication et Civilisation, 2003.

LARDELLIER P., Opéra bouffe. Une anthropologie gourmande de nos modes alimentaires, Cormelles-le-Royal, EMS : collection Societing, 2011.

LARDELLIER P., Nos modes, nos mythes, nos rites. Le social, entre sens et sensible, Cormelles-le-Royal, Éditions EMS : collection Societing, 2013.

LARDELLIER P., Risques, rites et plaisirs alimentaires, Cormelles-le-Royal, Éditions EMS : collection Societing, 2013.

MAAREK P., Communication et marketing de l'homme politique, Paris, Lexis Nexis : collection Carré Droit, 2007.

SANSOT P., Le rugby est une fête, le tennis non plus, Paris, Petite Bibliothèque Payot $n^{\circ} 420,2005$.

Résumé : Cet article se propose d'analyser la façon spécifique dont les valeurs propres à un sport collectif (en l'occurrence le rugby à XV) peuvent présenter un caractère pertinent et éclairant pour d'autres champs de l'activité humaine, qu'il s'agisse de la vie politique ou de la vie en entreprise. Il entend également montrer comment ces valeurs emblématiques peuvent être détournées et mises au service d'objectifs politiques, entrepreneuriaux et managériaux. 
Mots-clés : Management, Communication politique, Marketing politique, Sociologie du sport, Instrumentalisation.

Abstract: This article aims to analyze the specific way to own a team sport values (ie rugby union) may be a relevant and illuminating character for other fields of human activity, it s'act of political or business life. It also seeks to show how these iconic values can be diverted and made to serve political objectives, entrepreneurial and managerial skills

Keywords: Management, Political Communication, Political Marketing, Sociology of Sport, Instrumentalisation. 